\title{
Médiévales
}

Langues, Textes, Histoire

61 | automne 2011

La chair des émotions

\section{Une histoire des émotions incarnées}

\section{Damien Boquet et Piroska Nagy}

\section{(2) OpenEdition}

Journals

Édition électronique

URL : https://journals.openedition.org/medievales/6249

DOI : 10.4000/medievales.6249

ISSN : $1777-5892$

\section{Éditeur}

Presses universitaires de Vincennes

\section{Édition imprimée}

Date de publication : 20 décembre 2011

Pagination : 5-24

ISBN : 978-2-84292-337-2

ISSN : 0751-2708

Référence électronique

Damien Boquet et Piroska Nagy, « Une histoire des émotions incarnées », Médiévales [En ligne], $61 \mid$ automne 2011, mis en ligne le 17 janvier 2012, consulté le 22 avril 2022. URL : http://

journals.openedition.org/medievales/6249; DOI : https://doi.org/10.4000/medievales.6249 


\section{Damien BOQUET \\ Piroska NAGY}

\section{UNE HISTOIRE DES ÉMOTIONS INCARNÉES}

Les émotions aussi ont une histoire ${ }^{1}$. Ce constat, qui peut sembler d'évidence, est pourtant une conquête récente ${ }^{2}$. Certes, il n'a pas fallu attendre la dernière décennie pour voir les historiens s'intéresser aux affects et à leurs manifestations sociales. Qu'il suffise ici d'évoquer les figures emblématiques d'un Jules Michelet qui se dit lui-même touché par «le don des larmes ${ }^{3}$ », d'un Johan Huizinga qui reconnut dans les derniers siècles du Moyen Âge les feux vespéraux d'une émotivité exacerbée ${ }^{4}$, d'un Georges Lefebvre qui donna corps à l'abstraite psychologie des foules ${ }^{5}$, ou d'un Lucien Febvre dont le célèbre appel «La sensibilité dans l'histoire» apparaît aujourd'hui encore comme un texte fondateur pour nombre d'historiens de l'émotion ${ }^{6}$. Pour autant, malgré ces précédents illustres et les études inspirées qui ont suivi, consacrées à la

1. Nous tenons à remercier la psychologue Anna Tcherkassof pour sa contribution à notre réflexion collective et ses éclairages précieux qui nous ont accompagnés tout au long de la rédaction de cette introduction. Voir son ouvrage de synthèse, particulièrement utile autour du thème de ce dossier, Les Émotions et leurs expressions, Grenoble, 2008.

2. Voir B. H. RosenweIn, «Worrying about Emotions in History », American Historical Review, 107 (2002), p. 821-845.

3. J. Michelet, Le Moyen Âge, Paris, 1969, p. 22.

4. J. Huizinga, L'Automne du Moyen Âge (1919), Paris, 2002.

5. G. Lefebvre, La Grande Peur (1932), suivie de Les Foules révolutionnaires (1934), Paris, 1988.

6. L. Febvre, «La sensibilité dans l'histoire: les courants collectifs de pensée et d'action», dans La Sensibilité dans l'homme et dans la nature, 10' Semaine Internationale de Synthèse, 7-12 juin 1938, Paris, 1943, p. 77-106. 
peur de la damnation ${ }^{7}$, à l'amour chevaleresque ${ }^{8}$, au rire monastique ${ }^{9}$, aux gestes de désespoir ${ }^{10}$ ou à l'expressivité émotionnelle du visage ${ }^{11}$, l'histoire de l'affectivité ne s'est pas constituée, jusqu'à très récemment, en un champ historiographique autonome. Il faut dire que la position ancillaire de l'émotion dans l'historiographie était déterminée par les conditions mêmes de l'intérêt qu'on lui portait. En effet, pensée avec les outils et les cadres de la psychologie de la fin du XIX ${ }^{\mathrm{e}}$ siècle, l'émotion s'éloigna du giron de la philosophie morale et de la théologie pour devenir un phénomène physiologique détrônant définitivement la passion et l'ancien «mouvement de l'âme» ${ }^{12}$. Érigé en donnée issue de l'observation scientifique, l'hiatus entre la raison et l'émotion, l'un des paradigmes les plus ambigus de l'anthropologie occidentale, s'en trouva renforcé. Aussi révolutionnaire que fût en son temps l'ouverture d'un Lucien Febvre ou d'un Robert Mandrou vers la «psychologie historique ${ }^{13}$, elle véhiculait dans son origine même - et comment aurait-il pu en être autrement? - le dogme de l'irrationalité des émotions. Ce n'est que dans la seconde moitié du $\mathrm{xx}^{\mathrm{e}}$ siècle que l'on a commencé à concevoir que l'émotion pût avoir un rapport apaisé, voire complice, avec la raison qui analyse et évalue. Cette remise en cause de l'exclusivité cognitive de la raison est aujourd'hui largement diffusée et vulgarisée, notamment parce que la révision scientifique faisait écho à une demande sociale en faveur d'une plus grande reconnaissance de la valeur et de l'intelligence des émotions. Des approches scientifiques variées ${ }^{14}$, venues de la

7. J. Delumeau, La Peur en Occident (XIV'-XVIII siècles): une cité assiégée, Paris, 1978.

8. G. Duby, Mâle Moyen Âge: de l'amour et autres essais, Paris, 1988.

9. J. Le GofF, «Rire au Moyen Âge», Cahiers du Centre de Recherches Historiques, 3 (1989), p. 1-14, et «Le rire dans les règles monastiques du haut Moyen Âge», dans M. Sot éd., Haut Moyen Âge. Culture, éducation et société. Études offertes à Pierre Riché, Paris, 1990, p. 93-104.

10. M. Barasch, Gestures of Despair in Medieval and Early Renaissance Art, New York, 1976.

11. J.-F. Courtine et C. HAROCHE, Histoire du visage. Exprimer et taire ses émotions, XVI ${ }^{e}$-début XIX siècle, Paris, 1988.

12. T. Dixon, From Passions to Emotions. The Creation of a Secular Psychological Category, Cambridge, 2003.

13. R. MANDROU, Introduction à la France moderne : essai de psychologie historique, Paris, 1961.

14. Pour une vue d'ensemble, voir R. Harré et W. G. Parrott éd., The Emotions. Social, Cultural, and Biological Dimensions, Londres, 1996; R. PLuTCHIк, Emotions and Life. Perspectives from Psychology, Biology, and Evolution, Washington, 2005. Plusieurs revues consacrées à l'étude des émotions dans une perspective interdisciplinaire ont été récemment créées: Emotion Review (http://emr.sagepub.com) ou Passions in Context (http://www.passionsincontext.de) accordent un intérêt certain à l'approche historique. Consultées le 12.04.2011. 
psychologie cognitive ${ }^{15}$, des neurosciences ${ }^{16}$ ou de la philosophie de l'esprit ${ }^{17}$, ont mis en lumière le rôle des émotions dans les choix qui nous conduisent à agir, dans l'évaluation des expériences vécues, dans la communication interpersonnelle, dans les apprentissages et l'acquisition de connaissances. Loin d'être des parasites de la rationalité, les émotions en sont les sentinelles : elles nous renseignent sur ce qui est conforme à nos valeurs et aux attendus sociaux. On parle à juste titre d'un changement épistémologique majeur pour qualifier cette reconnaissance nouvelle de la qualité cognitive et de la rationalité morale des émotions, même s'il reste à en mesurer l'emprise véritable dans les sociétés contemporaines et à replacer les ruptures perçues dans le temps long d'une histoire des passions et des affects, dont la qualité morale fut longtemps admise.

\section{Quelle histoire? quelles émotions?}

Ainsi, le fondement de l'histoire des émotions réside dans la prise en considération par l'historien du nouveau paradigme de la cognition et dans le dialogue que celle-ci implique avec les sciences de l'émotion. Si cette histoire peut apparaître comme l'application tardive d'un volet du programme des Annales, rattaché au projet cher à Lucien Febvre de «psychologie historique» et à la nécessité de considérer au préalable «les derniers résultats acquis par le labeur critique et positif de nos voisins les psychologues ${ }^{18}{ }^{»}$, sa mise en œuvre est réalisée aujourd'hui sur des bases épistémologiques foncièrement différentes. Commençons par traiter la question des conditions du dialogue avec «nos voisins les psychologues ». Car, disons-le d'emblée, la diversité des présupposés et des méthodes d'approche de l'émotion cognitive se mesure à l'aune de la plasticité et de l'indéfinition de la notion même. L'émotion semble avoir conquis sa place dans le discours scientifique tout récemment, en désignant ce qui n'entrait pas dans la Raison universelle victorieuse de la modernité, voire tout ce qui en était exclu. Nous avons dressé un panorama de ces approches dans l'introduction au Sujet

15. Dans une production extrêmement abondante, voir par exemple N. FrIJDA, The Emotions, Cambridge, 1986; A. Ortony, G. Clore et A. Collins, The Cognitive Structure of Emotions, Cambridge, 1988; K. T. Scherer, A. Schorr et T. Johnstone, Appraisal Processes in Emotion, Oxford, 2001 ; P. LIVET, Émotions et rationalité morale, Paris, 2002 ou K. R. SCHERER, «Trends and Development: Research on Emotions », Social Science Information, 4 (2005), p. 695-729.

16. Voir A. Damasio, L'Erreur de Descartes. La raison des émotions, Paris, 1995 ; ID., Spinoza avait raison. Joie et tristesse : le cerveau des émotions, Paris, 2003 ou J. Ledoux, Le Cerveau des émotions : les mystérieux fondements de notre vie émotionnelle, Paris, 2005.

17. Voir notamment R. DE SousA, The Rationality and the Emotions, Cambridge (Mass.), 1987; R. C. Solomon, The Passions. Emotions and the Meaning of Life, Indianapolis, 1993 ou encore J. Elster, Alchemies of the Mind: Rationality and the Emotions, Cambridge, 1999.

18. L. FebVRe, «La sensibilité...», p. 97. 
des émotions au Moyen Âge ${ }^{19}$, en soulignant les deux grandes orientations dont beaucoup de scientifiques font aujourd'hui la synthèse ${ }^{20}$. D'un côté, une approche socioconstructiviste met l'accent sur les constructions culturelles qui déterminent les modalités de reconnaissance et d'expression de l'émotion, pour soi et en société, tout en la percevant comme une structure psychologique. D'un autre côté, une approche universaliste tend à induire des déterminismes biologiques de l'émotion des règles psychologiques et comportementales universelles. Bien entendu, on rencontre ces orientations déclinées à différents degrés, depuis un culturalisme strict, où la notion d'émotion est elle-même pensée comme un concept culturel, jusqu'au biologisme le plus obtus qui n'accorde que la portion congrue aux normes sociales et culturelles ${ }^{21}$.

Dans ce paysage, on ne saurait trop insister sur la spécificité de la matière historique, qui est comme un manteau tissé de fils de significations culturelles. L'émotion en tant qu'objet historique n'est en rien «l'émotion psychologique» puisqu'elle est une construction médiatisée par le document et ses modalités d'élaboration. Ainsi, les théories contemporaines qui ramènent les émotions et leurs expressions sociales à une mécanique fixe et universelle, régie par les impératifs de «l'homme neuronal ${ }^{22}$, sont incompatibles avec la démarche historique car elles nient de fait l'historicité des émotions, ou bien la réduisent à de simples effets de mise en scène. En revanche, nous admettons plusieurs degrés de constructivisme, et notamment que derrière ce que nous nommons peur, joie ou colère puissent se trouver des expériences partagées, des dispositions affectives universelles. Dans un rapport plus immédiat avec nos préoccupations, nous pouvons bien concevoir que l'émotion relève d'une structure cognitive et morale, tout en reconnaissant l'ascendant de la culture, des transmissions et des apprentissages, pour façonner les ressentis individuels et les usages sociaux, distinctement selon les temps et les environnements, les sociétés et les communautés ${ }^{23}$. Certainement, l'une des limitations induites par

19. Voir D. Boquet et P. NAGY, «Pour une histoire des émotions. L'historien face aux questions contemporaines», dans D. Boquet et P. NAGY éd., Le Sujet des émotions au Moyen Âge, Paris, 2009, p. 15-52 et A. Piolat et R. BAnNour, «Émotions et affects. Contribution de la psychologie cognitive», ibid., p. 53-84.

20. Voir en ce sens R. DE SousA, «À qui appartiennent les émotions ?», Critique, 625-626 (1999) ( Penser les émotions»), p. 497-498.

21. Voir ici par exemple les travaux du psychologue américain Paul Ekman. Pour en saisir l'orientation, il n'y a pas de meilleure introduction que son site: http://www.paulekman.com (consulté le 22.02.2011). Plus éloquent encore, voir le site de Claude Touzet: http://www.sciences-cognitives. org/ (consulté le 18.04.2011).

22. Voir F. WolfF, Notre humanité. D’Aristote aux neurosciences, Paris, 2010, p. 123-157. L'expression vient de J.-P. Changeux, L'Homme neuronal, Paris, 1983.

23. W. REDDY, «Against Constructionism. The Historical Ethnography of Emotions », Current Anthropology, 38/3 (1997), p. 329. 
ce constructivisme relatif tient dans le fait que l' "émotion psychologique» idéalement visée restera à jamais inaccessible à l'historien qui doit dès lors se satisfaire - mais la tâche est déjà immense ! - de mettre au jour ses représentations et codifications.

Pour l'historien, l'émotion performée ou figurée dans les sources est donc un événement culturel. Dès lors, l'histoire de l'émotion relève de l'anthropologie culturelle. Cependant, nous devons être particulièrement sensibles à l'emprise du prisme occidental dans l'élaboration savante et la perception sociale de ce que nous nommons émotion. Si un dialogue peut s'engager à partir de cet outillage intellectuel avec certaines cultures - comme le montrent par exemple les riches travaux portant sur l'histoire des émotions en Chine ${ }^{24}-$, d'autres cultures en revanche ignorent jusqu'au concept même d'émotion ${ }^{25}$, ce qui ne veut pas dire bien sûr que leurs membres ne la vivent pas. Nous apprenons des ethnologues qui se sont intéressés avant les historiens au rôle de l'affectivité dans la construction de la personne et des sociétés que les anthropologies non occidentales, pour autant que nous y ayons accès, ne localisent pas, ne structurent pas, ne vivent pas les ressentis affectifs de manière analogue à la nôtre ${ }^{26}$.

En conséquence, la médiation de la source historique et les formes culturelles dans lesquelles l'émotion prend forme ne sauraient représenter un masque

24. Voir avant tout les travaux de Paolo Santangelo, et son projet de recherche: http://w3.uniroma1.it/Santangelo/emotions.htm (consulté le 01.05.2011); P. SANTANGelo et U. MidDEndoRf éd., From Skin to Heart. Perceptions of Bodily Sensations and Emotions in Traditional Chinese Culture, Wiesbaden, 2006; P. SAnTANGelo et D. Guida éd., Love, Hatred, and Other Passions : Questions and Themes on Emotions in Chinese Civilization, Leyde, 2006; P. SANTANGElo éd., Passioni d'Oriente. Eros ed emozioni nelle civiltà asiatiche. Sezione Asia Orientale. Atti del Convegno, Roma, La Sapienza, 29-31 maggio 2003, dans Suppl.n. 4, Rivista di Studi Orientali, LXXVIII, Rome, 2007 [2008]; sur l'histoire chinoise ancienne et médiévale de la pensée des émotions, voir les travaux de Curie Virag, notamment C. VIRAG, «Emotion, Knowledge, and the Reconfigured Self in the TangSong Transition», dans P. Santangelo et D. Guida éd., Love, Hatred, and Other Passions..., p. 166179; EAD., «Emotions and Human Agency in the Thought of Zhu Xi», Journal of Song Yuan Studies, 37 (2007), p. 49-88 - particulièrement la définition de la notion de qing, p. 52-53; EAD., «Early Confucian Perspectives on Emotions », dans V. SHEN et K. SHun éd., Handbook of Confucian Thought, New York (à paraître) ; voir enfin son projet de livre consultable sur le site du Collegium Budapest Institute for Advanced Study, http://www.colbud.hu/fellows/current.shtml (consulté le 15.05.2011).

25. C. Lutz, «The Domain of Emotion Words on Ifaluk», dans R. HARré éd., The Social Construction of Emotions, Oxford, 1986, p. 267-288.

26. Même si nous n'y avons accès presque exclusivement qu'au travers des travaux des anthropologues occidentaux. Ceux-ci sont néanmoins nombreux et désormais classiques : voir avant tout R. Harré éd., The Social Construction..., et M. Z. Rosaldo, Knowledge and Passion: Ilongot Notions of Self and Social Life, Cambridge, 1980; L. ABu-Lughod, Veiled Sentiments: Honor and Poetry in a Bedouin Society, Berkeley, 1986; C. LuTZ, Unnatural Emotions : Everyday Sentiments on a Micronesian Atoll and their Challenge to Western Theory, Chicago, 1988; O. M. LyNCH éd., Divine Passions. The Social Construction of Emotion in India, Berkeley-Los Angeles-Oxford, 1990. 
occultant la profondeur psychologique d'une émotion «authentique», pour la simple et bonne raison que l'émotion n'a pas de réalité saisissable pour l'historien en dehors du dispositif culturel qui la produit. Le champ historique possède donc ses spécificités dans l'étude des émotions par rapport à la psychologie, qui ne sont en rien des limitations pour l'enquête. De cette façon, dans sa pratique même, l'historien envisagera l'émotion du psychologue non pas comme une catégorie scientifique qu'il lui reviendrait de projeter sur les documents du passé, mais pour son potentiel heuristique, pour sa capacité à mettre en lumière la contingence de l'ancien prisme de l'irrationalité des émotions et à enrichir le questionnement dans une perspective généalogique. L'émotion se comprend ainsi au cœur des processus socioculturels de l'Occident, à la fois à travers la relation qu'elle entretient avec la construction sociale du corps, et par son importance dans les événements, les changements, les transformations. Elle est définie conjointement par sa dimension relationnelle (horizontale, synchronique) et par sa dimension culturelle (verticale, diachronique).

\section{Écrire l'histoire des émotions médiévales}

En l'espace d'une décennie, l'histoire des émotions est passée de l'âge des manifestes ${ }^{27}$ à celui des programmes institutionnels ${ }^{28}$ : tout se passe comme si après une longue phase d'éveil, l'histoire des émotions avait connu une maturation accélérée ${ }^{29}$, dont on ne peut que se réjouir mais dont les modalités, y compris les plus matérielles, doivent également être questionnées. Ce n'est pas le lieu ici de lancer la réflexion sur la portée des cadres institutionnels dans l'orientation des champs de la recherche. En revanche, il peut être utile, en raison

27. Voir en particulier B. H. Rosenwein, «Worrying about Emotions in History» et, bien en amont, P. N. Stearns et C. Z. Stearns, «Emotionology. Clarifying the History of Emotions and Emotional Standards », American Historical Review, 90 (1985), p. 813-836.

28. Voir les principaux programmes présentés dans la rubrique «Liens» de notre carnet de recherche en ligne (http://emma.hypotheses.org).

29. Du moins peut-on l'affirmer pour ce qui concerne l'Occident médiéval où une vue synthétique des évolutions devient possible. En revanche, pour ses voisins - Byzance et le monde musulman -, les études sont encore rares. Pour Byzance, voir par exemple É. Limousin, «Les émotions de l'empereur byzantin», dans, D. Boquet et P. NAGy éd., Politiques des émotions au Moyen Âge, Florence, 2010, p. 33-48; M. HINTERBERGER, «Tränen in der Byzantinischen Literatur. Ein Beitrag zur Geschichte der Emotionen », Jahrbuch der Österreichischen Byzantinistik, 56 (2006), p. 27-51; ID., «Emotions in Byzantium», dans L. James éd., A Companion to Byzantium, Oxford, 2010, chap. 10, p. 123-134. Pour le monde musulman, un important projet de recherche financé par l'European Research Council porte sur un sujet comparatif, qui inclut nécessairement les émotions : Subjectivity and Selfhood in the Arabic and Latin Traditions. Voir le programme du séminaire organisé à ce sujet sur http://emma.hypotheses.org/1257 (consulté le 30.04.2011). 
de ce succès même, de préciser à nouveau la nature et le périmètre de l'émotion comme objet historique.

L'histoire des phénomènes affectifs a été d'emblée dénommée en adoptant le vocable anglais emotion, dans la mesure où les premiers appels en faveur d'une history of emotions sont venus des États-Unis ${ }^{30}$, et en raison, bien entendu, de la domination de l'anglais dans l'usage académique. En anglais, emotion est un terme à spectre sémantique large, qui englobe son équivalent français, tout en le débordant, notamment du côté du sentiment. Le même rapport, qu'on pourrait qualifier d'équivalence élargie, existe avec l'espagnol emoción ou l'italien emozióne. En allemand, on note également une tendance forte chez les spécialistes de l'émotion à utiliser le décalque Emotion à la place de termes plus traditionnels tels Gefühl, Empfindung ou Affekt ${ }^{31}$. Si l'histoire des émotions avait été essentiellement francophone, elle aurait probablement été dénommée «histoire de l'affectivité», couvrant un large domaine allant des dispositions affectives et des traits de caractère aux sentiments durables et aux émotions qui, en français, désignent clairement un mouvement psychique bref, le plus souvent reflété par le corps. Pour le Moyen Âge, l'usage du mot émotion peut sembler anachronique, voire présentiste, puisque le terme, qui apparaît au $\mathrm{XvI}^{\mathrm{e}}$ siècle en français, ne prend qu'au XIX ${ }^{\mathrm{e}}$ siècle son sens actuel de catégorie psychologique unifiée et moralement neutre ${ }^{32}$. Il reste que pour désigner de façon générique ce champ historiographique, le terme d'émotion demeure sans doute le plus approprié, en raison du consensus international à ce sujet, de sa commodité d'usage dans les principales langues académiques, mais surtout parce qu'il favorise une approche globale de l'affectivité qui résiste à l'émiettement de l'objet, ce mal de l'historiographie contemporaine. Pour autant, on se gardera de faire de l'émotion un artefact psychologique tout comme on échappera à l'illusion d'espérer la contenir dans une définition catégorielle. Si l'objet visé par l'enquête est prioritairement l'émotion comprise dans son acception traditionnelle en français de phénomène affectif transitoire ${ }^{33}$, il ne peut être question de lui imposer

30. Voir C. Z. Stearns et P. N. Stearns, Emotion and Social Change. Toward a New Psychohistory, New York-Londres, 1988.

31. Voir par exemple B. FREUDENBERG éd., «Furor, Zorn, Irance». Inter-disziplinäre Sichtweisen auf mittelalterliche Emotionen, Berlin, 2009 (Das Mittelalter, 14/1); C. Newmark, Passion - Affekt - Gefühl. Philosophische Theorien der Emotionen zwischen Aristoteles und Kant, Hambourg, 2008 (Paradeigmata, 29); E. Косн, Trauer und Identität. Inszenierungen von Emotionen in der deutschen Literatur des Mittelalters, Berlin/New York, 2006 (Trends in Medieval Philology, 8); J. EMING, Emotion und Expression. Untersuchungen zu deutschen und französischen Liebes- und Abenteuerromanen des 12. bis 16. Jahrhunderts, Berlin/New York, 2006.

32. Voir T. Dixon, From Passions to Emotions...

33. Dans Le Sujet des émotions au Moyen Âge, nous proposons la définition heuristique suivante de l'émotion : «territoire des phénomènes affectifs transitoires de plaisir-déplaisir, de désir-répulsion, 
des frontières exclusives. Ainsi, on n'isolera pas l'émotion du sensible en amont, ni du sentiment en aval, liés qu'ils sont par la dynamique même du phénomène affectif. Cette acception ouverte de l'émotion-affectivité est encouragée par la démarche historique elle-même: construire une histoire des émotions, c'est en effet étudier les conceptions et usages du registre affectif dans un corpus en le lisant à l'aune des normes et pratiques d'écriture des sources, tout en maintenant le dialogue, condition indispensable du sens produit, entre les catégories actuelles et les catégories anciennes.

En outre, afin de couper court à cette autre illusion d'une histoire des émotions qui sortirait tout armée des lexiques et des concepts médiévaux, rappelons qu'on serait bien en peine de substituer à l'émotion moderne une notion médiévale stable et générale, et certainement pas la «passion» qui n'a pris un ascendant que tardivement, à partir du XIII ${ }^{\mathrm{e}}$ siècle, après de longs siècles de domination des «affects» et autres «mouvements de l'âme». Comme l'a montré Thomas Dixon, jusqu' au XIX ${ }^{\mathrm{e}}$ siècle, le registre affectif, loin de se contracter sous la forme d'une catégorie psychique circonscrite, s'exprime à de multiples niveaux de la dynamique du vivant et de l'écheveau des puissances de l'âme: passio, affectus, affectio, motus, inclinatio sont autant de dispositions, tantôt spécifiques, tantôt semblables, qui tendent l'âme de désirs et de répulsions, de plaisirs et de souffrances, dans un rapport étroit avec le corps et les puissances de la rationalité. Il est donc indispensable de suivre chacun de ces rameaux généalogiques, en y incluant bien entendu la branche directe de l'emovere, afin de reconstruire le jeu serré des réseaux sémantiques et conceptuels ${ }^{34}$. Mais jamais la plongée dans la logique intrinsèque des sources n'abolira le lieu et le sujet originels du questionnement ${ }^{35}$. C'est pourquoi une histoire de l'émotion est légitime. C'est pourquoi aussi celle-ci ne saurait être la somme des histoires de l'affectus, de la passio, du sentimentum, de l'esmouvance, etc. À l'inverse, elle ne sera jamais l'histoire d'un déterminé biologique ou psychologique dont il conviendrait de traquer les avatars dans le passé.

L'émotion est objet d'histoire dans la mesure où le passé peut être mis à l'épreuve des problématisations du présent. Nous proposons de mettre en œuvre cette histoire-là selon deux approches concertées que l'on peut concevoir comme deux cercles concentriques, ou mieux comme deux régimes d'analyse: le premier

dans l'infinie diversité de leurs configurations, qui sont associés par le sujet à l'évaluation de la situation vécue», voir D. BoQuet et P. NAGY, «Pour une histoire...», p. 47.

34. Voir en ce sens D. BoQUET, «Les mots avant les choses: mystique cistercienne et anthropologie historique de l'affectivité», dans A. Dierkens et B. BEyer DE RyKe éd., Mystique, la passion de l'Un. De l'Antiquité à nos jours, Actes du colloque international de Bruxelles, 9-11 décembre 2004, Bruxelles, 2005 (Problèmes d'histoire des religions, 15), p. 109-119.

35. Voir D. Boquet et P. NAGY, «Émotions historiques, émotions historiennes », Écrire l'histoire, 2 (2008), p. 15-26. 
serait général et descriptif; le second, plus spécifique et dynamique. Au sens large, l'histoire des émotions est une histoire de l'anthropologie affective des sociétés d'antan, donc des théories et représentations des phénomènes affectifs, conjuguée à une anthropologie historique de l'affectivité, entendue comme l'histoire de l'ensemble des façons de vivre et d'user de l'affect en société. À un second niveau, l'émotion dont on fait l'histoire peut être conçue comme une tension continue de l'affectivité. Dit en termes plus historiens, l'émotion est ce qui fait événement dans le flux de la vie affective. Pour envisager cette disposition spécifique, il peut être intéressant de s'inspirer des lectures prototypiques de l'émotion proposées par la psychologie cognitive ${ }^{36}$. En linguistique et dans les sciences cognitives, les prototypes représentent les éléments les plus communément reconnus d'une catégorie. En ce sens, plusieurs composantes nous paraissent particulièrement caractériser l'émotion en son acception restreinte: l'implication corporelle, les dispositions cognitives, l'évaluation morale et l'orientation vers l'action. D'ailleurs, pour l'historien, la prise en considération de l'approche componentielle de l'émotion s'avère d'autant plus opératoire qu'elle fait écho aux théories antiques, notamment à Aristote qui identifiait déjà quatre composantes dans la passion: une évaluation positive ou négative pour le sujet, une sensation agréable ou désagréable associée à l'évaluation, une motivation vers l'action et enfin un changement corporel ${ }^{37}$. Suivant cette orientation actuelle et historique, on reconnaîtra une émotion lorsque ces caractéristiques convergent dans un discours affectif (qu'il soit de nature textuelle ou figurée). Bien entendu, il convient d'adapter cette démarche empirique en fonction du corpus documentaire abordé. Ainsi, il n'est pas question d'exiger que toute la structure soit en place pour parler d'émotion, ce qui reviendrait une nouvelle fois à réifier la notion. L'émotion se manifeste comme un processus, et c'est dans la dynamique même de cet avènement qu' elle est un agent de la transformation historique. L'historienanthropologue américain William Reddy a appelé cet «agir» de l'émotion emotive, qu'on peut traduire par émotif, par lequel il nomme l'émotion qui s'exprime, en paroles ou par gestes. L'émotif - calqué sur le «performatif» de John Austin ${ }^{38}$ - est un énoncé particulier, qui transforme l'état affectif de la personne qui le dit ou fait, ainsi que celui de son interlocuteur. Car un émotif, geste ou énoncé émotionnel, ne peut jamais être précis selon W. Reddy: en

36. B. Rimé, «Les émotions médiévales. Réflexions psychologiques», dans D. BoQuET et P. NAGY éd., Politiques des émotions..., p. 311-312.

37. Аristote, Rhétorique, 2, chap. 1-11, 1377b-1388b, Paris, 1991, p. 181-232. Pour une présentation de la théorie aristotélicienne des passions et de son écho dans la psychologie cognitive, voir S. KnuUtTila, Emotions in Ancient and Medieval Philosophy, Oxford, 2004, p. 24-47 (plus particulièrement p. 32-33).

38. J. L. Austin, Quand dire, c'est faire, Paris, 1970. 
désignant une réalité affective, il la change déjà. Il y a un décalage inévitable entre l'état affectif et son expression. Si la notion d'émotif semble à première vue difficilement utilisable pour l'historien, bien qu'il puisse en entrevoir l'utilité pour l'anthropologue ou le sociologue, elle ouvre de nouvelles perspectives vers une meilleure compréhension du processus émotionnel et de sa force historique. L'émotion historique, telle qu'elle est construite et transmise par les sources, renvoie à un frémissement de la chair, à jamais perdue. Une des objections les plus fréquentes faites à une telle histoire lui reproche précisément cette fugacité de la trace de l'émotion, qui rendrait toute saisie improbable et invérifiable. Pourtant, sa tension dynamique reste présente, au creux des sources, comme un tressaillement mystérieux. En analysant, en écrivant, c'est bien ce que l'historien tente de saisir et de restituer, cette chair des émotions qui palpite dans la chair des textes: la dynamique entre le dit et le dicible, l'attendu et l'indicible, les effets prévisibles d'un geste et sa nécessité ou sa légitimation dans une situation ${ }^{39}$.

Il reste à valider la pertinence heuristique de chacune de ces propositions, dans un cadre large lorsque c'est possible et de façon impérative à l'échelle de chaque dossier considéré. Par exemple, le lien organique entre l'anthropologie des passions de l'âme et la théologie morale des vices et des vertus tout au long du Moyen Âge est un argument fort pour valider l'orientation axiologique de l'émotion. L'objectif de ces études consacrées aux rapports entre corps et émotions est donc d'approfondir l'exploration d'un des axes de l'émotion historique, engageant ainsi une nouvelle étape de la réflexion.

\section{La chair des émotions}

\section{Techniques des émotions}

On pâlit, on rougit, on tremble, on sent notre cœur palpiter, notre voix tonne: l'émotion se dit par le corps. Elle est identifiée par sa dynamique, perceptible dans et sur le corps, qu'il s'agisse du nôtre ou de celui d'autrui - d'une manière plus évidente, plus fréquente que pour les sentiments ou les dispositions affectives. Pour cette raison, certains psychologues considèrent qu'on peut lire l'émotion indépendamment des cultures, à partir de l'expression du visage ${ }^{40}$. Certes, il existe des mécanismes physiques automatiques qui mobilisent des marqueurs corporels de l'émotion - qui n'a pas pleuré en épluchant un oignon ou

39. On peut citer en ce sens $\mathrm{S}$. WAHNICH, «Constellation du sensible, l’homme, le révolutionnaire, l'historien», dans EAD., Les Émotions, la Révolution française et le présent, Paris, 2009, p. 11-58 (surtout p. 30-36) ; G. Duby, L'Histoire continue, Paris, 1989, p. 78; A. FARge, Le Goût de l'archive, Paris, 1989, p. 42.

40. Voir par exemple P. EKMAn, Emotions Revealed. Recognizing Faces and Feelings to Improve Communication and Emotional Life, New York, 2007. 
succombé au fou rire sous l'effet des chatouilles ?-, mais ceux-ci ne sont pas plus l'émotion que l'œuf et le pigment sur la toile ne sont la valeur artistique d'une peinture. D'évidence, les émotions ont une matière et un support corporels qui en conditionnent le ressenti et l'expression, mais ni la biologie ni la dynamique des muscles ne produisent en soi rien qui puisse être appelé émotion, laquelle est précisément l'objet culturel tout entier contenu dans le signe et l'échange. D'ailleurs, rappelons que la correspondance n'est ni nécessaire, ni exclusive entre l'émotion et sa traduction corporelle, comme le croient ces néophysiognomonistes qui cherchent à saisir l'émotion à partir des expressions faciales, voire à la «lire» sur le corps comme le propose par exemple la synergologie ${ }^{41}$. Ces lectures mécanistes s'enracinent dans une tendance lourde des sociétés contemporaines, nourrie d'une vision étroitement scientifique du monde, qui conduit au discrédit du langage et du lien socialement construit et promeut la quête d'une vérité immanente et immédiate, saisissable dans l'être biologique.

On pâlit, on rougit, on rit ou on pleure dans telle ou telle situation. L'anthropologue et l'historien savent bien la force des normes culturelles qui répriment, modèlent, modulent les expressions émotives. Dans tous les cas, nous insérons les signes corporels et les ressentis qui les accompagnent dans un tissu de significations culturelles préétabli, pour ensuite élaborer cette configuration affectivo-corporelle qu'est l'émotion. L'émotion physiologique peut «prendre » des noms, des contenus et des valences bien différents selon les cultures voire les situations, et souvent, en éprouvant l'émotion, on ne sait pas d'emblée de quelle émotion il s'agit: juste «je suis ému/e». Sous un autre angle, éprouver une émotion dans une situation donnée n'est pas obligatoire, mais dépend du contexte, large et étroit: devant s'adresser à un public nombreux, tel se sentira tétanisé et tel autre restera placide, ou ressentira même des frissons de plaisir. De même, aucun événement physiologique n'est univoque: un tel, habitant des villes, tremblera de peur devant le lion; tel autre, chasseur aguerri, pourra trembler d'excitation. Lorsque nous vivons et échangeons, il se passe «quelque chose de corporel», qui interagit avec notre histoire et en oriente le sens. Mais l'événement produit est entièrement culturel, y compris dans la façon d'isoler et de nommer telle séquence, telle fonction. L'émotion est l'un de ces dispositifs par lesquels dialoguent le corps, le monde reçu et l'expérience. Il existe des techniques de l'émotion, façonnées par les actes traditionnels efficaces dont parle Marcel Mauss, comme il existe des techniques du corps ${ }^{42}$. C'est même précisément le lien entre émotion et corps qu'on doit considérer comme une

41. Voir www.synergologie.org (consulté le 21.02.2011). Sur la dimension foncièrement relationnelle de l'expression des émotions, voir A. TCHERKASSOF, Les Émotions...

42. M. MAuss, «Les techniques du corps », Journal de psychologie, 32 (1936), publié dans ID., Sociologie et anthropologie, Paris, 2004, p. 362-386 (ici p. 371-372). 
donnée de la culture occidentale, et non de la «nature» universelle: aucun déterminisme physiologique «n'oblige» à trembler «de peur», à rougir «de colère» ou «de honte».

\section{Les mouvements de l'âme et leurs expressions corporelles}

Bien en amont de notre monde contemporain, la pensée occidentale a lié l'émotion au corps depuis l'Antiquité. Mais, à la différence de la pensée scientifique actuelle qui tend à identifier l'être vrai des émotions à leurs manifestations corporelles ou neuronales, pour les anciens ces manifestations étaient les signes visibles de mouvements qui avaient leur origine dans l'âme.

Pour Platon comme pour Aristote, les émotions ont une valeur ontologique, ce sont des puissances constitutives de la nature humaine qu'on ne peut donc anéantir ou extirper. En plaçant les émotions du côté de l'âme irrationnelle et appétitive, Platon lie déjà leur destin aux changements corporels ${ }^{43}$, mais c'est l'hylémorphisme d'Aristote qui fait des manifestations corporelles une composante propre de la passion. Ainsi, pour Aristote, le changement corporel semble être la cause matérielle de l'émotion tandis que les autres composantes (l'évaluation, le ressenti, la tendance à l'action) en sont la cause formelle: «il est évident que les émotions $(\pi \alpha \dot{\theta} \theta \eta)$ sont des formes engagées dans la matière ${ }^{44}$. Une disposition physiologique peut influer sur l'émotion, tout comme, en sens inverse, l'émotion ressentie s'accompagne de changements physiques. Le stoïcisme, dont on sait toute l'influence qu'il aura sur l'anthropologie chrétienne, fait la synthèse de ces deux orientations : les émotions sont des mouvements de l'âme qui entraînent des changements corporels. Néanmoins, un auteur comme Sénèque refuse d'accorder au corps une causalité efficace sur l'émotion. Celui qui a peur blêmira sans doute, voire tremblera de tous ses membres; cependant, ni la pâleur, ni le tremblement ne sont par eux-mêmes l'émotion (affectus) puisqu'ils peuvent n'être que de simples réflexes corporels (pulsus corporis) ${ }^{45}$. C'est une position semblable que l'on trouve chez les théologiens chrétiens de la génération de saint Augustin. Ce dernier conçoit que les émotions s'accompagnent de manifestations corporelles mais elles demeurent des mouvements exclusivement de l'âme ${ }^{46}$. Dans l'anthropologie moniste d'Augustin, le corps n'est jamais ce qui dicte le mouvement de l'âme. Et si l'on peut parfois en avoir l'impression, ce

43. Platon, République, Paris, 1966, 438d-445e, p. 191-199 ou, sur le rattachement des passions à la vie corporelle, Timée, Paris, 1969, 69a-70a, p. 447.

44. Aristote, De l'âme, 403a25, Paris, 1934, p. 10. Voir l'analyse de S. Knuuttila, Emotions in Ancient and Medieval Philosophy, p. 32-34.

45. SÉnÈQue, De la colère, livre II, IV, 1, Paris, 1971, p. 31.

46. Voir la définition d'inspiration stoïcienne de l'affectio: «Affectiones nostrae motus animorum sunt. Laetitia, animi diffusio; tristitia, animi contractio; cupiditas, animi progressio; 
n'est pas le corps qui agit mais la «chair», comprise ici comme la corporéité contaminée par une volonté viciée, «car la volonté est en tous ces mouvements, ou plutôt tous ces mouvements ne sont rien d'autre que des volontés ${ }^{47}$. Il reste que la connivence entre la chair et le corps, qui marque si fortement la pensée patristique, ne fait que renforcer la porosité entre la dynamique corporelle et la dynamique émotionnelle qui est elle-même très tôt tiraillée entre le vice et la vertu.

En outre, le compagnonnage étroit entre le corps et les émotions se trouve entretenu voire renforcé dans l'héritage de la pensée médicale antique. En effet, dans la médecine galénique, les émotions - passiones ou motus animae - figurent parmi les six causes ou facteurs non naturels qui influent sur la santé: l'air et l'environnement, la nourriture et la boisson, le sommeil et la veille, le mouvement et le repos, l'évacuation et la réplétion, enfin les passions de l'âme. Ces facteurs ou causes apparaissent déjà dans les écrits hippocratiques, puis de façon plus systématique chez Galien, pour devenir véritablement une catégorie au Moyen Âge, dans l'Isagoge de Hunayn Ibn Ishaq (Johannitius) au Ix' siècle. Leur nombre est fixé à six au $\mathrm{X}^{\mathrm{e}}$ siècle par Hali Abbas ${ }^{48}$ dont la somme médicale, le Kitāa kāmil aş-şinā 'aţibbìya', grâce à la traduction qu'en fait Constantin l'Africain vers 1080 sous le titre Pantegni, participe activement à la renaissance de la passion psychosomatique au $\mathrm{XII}^{\mathrm{e}}$ siècle dans le monde latin, bien avant le grand réveil aristotélicien ${ }^{49}$. Ainsi, dans la Theorica, première partie du Pantegni, Constantin

timor, animi fuga est.» Augustin, In Johannis Evangelium tractatus CXXIV, éd. A. MeYER, Turnhout, 1954 (CCSL, 36), tract. 46, 8, p. 403.

47. «Voluntas est quippe in omnibus; immo omnes nihil aliud quam voluntates sunt.» Augustin, La Cité de Dieu, t. II-1, XIV, VI, Paris, 1994 (Nouvelle Bibliothèque Augustinienne, 4, 1), p. 368369.

48. Pour une bonne synthèse, voir L. García-BALleSter, «On the Origin of the "Six Non-Natural Things" in Galen», dans ID., Galen and Galenism: Theory and Medical Practice from Antiquity to the European Renaissance, Aldershot, 2002 (Variorum, IV), p. 105-115, et auparavant S. JARCHO, «Galen's Six Non-Naturals», Bulletin of the History of Medicine, 44 (1970), p. 372-377; P. H. NIEBYL, «The Non-Naturals», Bulletin of the History of Medicine, 45 (1971), p. 486-492; L. J. RATHER, «The "Six Things Non-Natural"», Clio Medica, 3 (1968), p. 337-347. Enfin, en rapport avec les émotions, voir maintenant la thèse de doctorat de Na'ama CohEn-HANEgBi, Accidents of the Soul: Physicians and Confessors on the Conception and Treatment of Emotions in Italy and Spain, late 12th-15th Centuries, Jérusalem, Hebrew University, 2011.

49. Voir par exemple l'emploi tout à fait intéressant du terme passio, tantôt associé au corps, tantôt à l'âme dans le De natura corporis et animae de Guillaume de Saint-Thierry (écrit vers 1140) qui emprunte une bonne part de sa matière au Pantegni. Sur cette présence de la passion médicale dans la pensée du cloître et de l'école au XII ${ }^{\mathrm{e}}$ siècle, voir M. LemoinE, «Les ambiguïtés de l'héritage médiéval: Guillaume de Saint-Thierry», dans B. Besnier, P.-F. Moreau et L. Renault éd., Les Passions antiques et médiévales, Paris, 2003, p. 297-308 et D. BoqueT, «Un nouvel ordre anthropologique au $\mathrm{XII}^{\mathrm{e}}$ siècle: réflexions autour de la physique du corps de Guillaume de SaintThierry », Cîteaux, 55 (2004), p. 5-20. 
relie les émotions ou accidents de l'âme au corps par la théorie des humeurs ${ }^{50}$ : les dysfonctionnements du système humoral provoquent des symptômes émotionnels associés et, à l'inverse, agir sur les émotions fait partie des remèdes contre le déséquilibre des humeurs. On retrouve le même type de relations entre les émotions et les affections physiques dans le De anima d'Avicenne ${ }^{51}$. Par la suite, les régimes de santé, genre médical répandu aux derniers siècles du Moyen Âge, prennent en compte les émotions bonnes ou mauvaises pour la santé, qu'il convient de rechercher ou, au contraire, d'éviter. À partir du XIV siècle, quand la structure même de ces traités se fonde sur les six non-naturels (et non plus sur les membres et les maladies, comme auparavant ${ }^{52}$, l'intérêt pour les émotions crô̂t considérablement ${ }^{53}$.

À partir de la fin du XII siècle, l'accès à toute l'œuvre d'Aristote, à de larges pans du savoir grec et byzantin, enfin et surtout à la médecine arabe, a accentué la tendance à relier corps et âme autour des émotions, en renforçant un processus dont la dynamique était endogène. Déjà, l'intense réflexion cistercienne sur les affectus avait annoncé le passage de l'anthropologie augustinienne foncièrement spiritualiste (le corps ne peut rien éprouver sans le secours de l'âme) vers une reconnaissance graduelle de la naturalité des émotions (c'est par l'affect que l'âme reçoit les signaux des sens) ${ }^{54}$. Dans les premières décennies du XIII ${ }^{\mathrm{e}}$ siècle, Jean de La Rochelle, maître de théologie franciscain de la première génération, fait de la passion l'un des modes d'union de l'âme et du corps dans sa Summa de anima ${ }^{55}$. Par là, il lui confère, comme l'a remarqué Alain Boureau, un statut actif qui débouche sur une nouvelle conception de la subjectivité ${ }^{56}$. Loin d'être une maladie de l'âme, la passion est désormais un trait constitutif de l'homme. Thomas d'Aquin, qui insère dans sa Somme théologique le premier traité

50. Voir Constantin l'Africain, Pantegni, Theorica, VI, 109-114, dans Omnia opera Ysaac, II, Lyon, 1515, f. 25v et S. KnuUtTila, Emotions in Ancient and Medieval Philosophy, p. 214-215.

51. S. Knuuttila, Emotions in Ancient and Medieval Philosophy, p. 222-225.

52. N. Cohen-Hanegbi, Accidents of the Soul..., p. 71; P. Gil-Sotres, L. Garcia Ballester, M. McVAugh, Regimen sanitatis ad regem aragonum. Arnaldi de Vilanova Opera Medica Omnia, vol. X.1, Barcelone, 1996, p. 513-559; M. Nicoud, Les Régimes de santé au Moyen Âge, Rome, 2007, p. 61-184.

53. Voir N. Cohen-Hanegbi, Accidents of the Soul..., chap. 2.

54. Voir D. Boquet, L'Ordre de l'affect au Moyen Âge. Autour de l'anthropologie affective d'Aelred de Rievaulx, Caen, 2005, p. 119-149.

55. Voir Jean de La Rochelle, Summa de anima, éd. J. Bougerol, Paris, 1995, partie I, section VI: «De anima quantum ad corpus», et VIII : «De passibilitate anime».

56. Voir A. Boureau, «Un sujet agité. Le statut nouveau des passions de l'âme au XIII siècle», dans D. Boquet et P. NAGy éd., Le Sujet des émotions..., p. 187-200. Erich Auerbach avait noté en son temps le tournant de l'activation de la passion, longtemps passive, qu'il avait situé plutôt au début de l'époque moderne, voir E. Auerbach, «De la passio aux passions» (1941), dans ID., Le Culte des passions au XVII siècle, Paris, 1998, p. 51-79. 
médiéval sur les passions, parachève cette évolution : «dans les passions de l'âme le mouvement même de la puissance appétitive est comme l'élément formel, et la modification organique, l'élément matériel. Il y a correspondance de l'un à l'autre. D'où une ressemblance entre les caractéristiques des mouvements de l'appétit et la modification physiologique qui s'ensuit ${ }^{57}{ }^{\prime}$. Revenue au XII ${ }^{\mathrm{e}}$ siècle dans les bagages de la pensée naturaliste, la passio, libérée de la charge négative qui lui avait été assignée par les stoïciens, absorbe une part de la dynamique spirituelle des affectus. En outre, cet intérêt pour la dimension psychosomatique de l'émotion prend place dans le contexte d'une réflexion théologique nouvelle qui met le rapport corps-âme au cœur de l'intelligibilité de l'homme, cela dans un raisonnement qu'on peut appeler psychologique à partir du milieu du XII ${ }^{\mathrm{e}}$ siècle, et qui a pour but principal l'étude de l'âme, de la psyché humaine. Se développe alors une nouvelle spiritualité, dont le De spiritu et anima, une compilation cistercienne datant de 1170 environ et très vite attribuée à Augustin, est un prototype et une référence pour les scolastiques. Ces réflexions, que l'on trouve notamment dans les commentaires des De anima d'Avicenne et d'Aristote, ainsi que dans les traités scolastiques qui en intègrent la connaissance, ne se limitent plus à une perspective sotériologique mais envisagent plus largement la constitution de l'homme dans une optique anthropologique.

Ainsi, l'interpénétration des discours sur l'émotion entre philosophie naturelle, médecine, spiritualité et théologie semble bien être propre à un moment historique. Comme le montre Na'ama Cohen-Hanegbi, aux XIV ${ }^{\mathrm{e}}-\mathrm{XV}^{\mathrm{e}}$ siècles, le développement professionnel concurrent de la médecine et de la théologie dans le cadre de l'institution universitaire sonne le glas d'une pensée holistique de l'homme où les régimes du corps et de l'âme recouraient de manière identique à l'émotion. Deux approches parallèles émergent, séparant de plus en plus médecine du corps et médecine de l'âme ${ }^{58}$.

\section{Des affects médiévaux chrétiens?}

La construction culturelle qui relie l'émotion et le corps est déterminée d'une manière particulière dans la civilisation de l'Occident chrétien médiéval, bâtie autour de la religion de l'incarnation de Dieu ${ }^{59}$. Ce poids apparent du christianisme dans la civilisation et dans l'historiographie du Moyen Âge occidental provient de l'emprise quasi exclusive de l'Église sur la culture écrite (et sur

57. Thomas D’Aquin, Somme théologique, II-1, 44.1, t. 2, Paris, 1984, p. 279.

58. N. Cohen-Hanegbi, Accidents of the Soul..., p. 283-294 et passim.

59. Voir l'exploration de ses implications dans D. Boquet et P. NAGY éd., Le Sujet des émotions..., à travers les contributions de E. Coccia, C. Casagrande et $\mathrm{S}$. Vecchio, mais aussi de P. Nagy et de B. d'Hainaut-Zveny. 
la production des images) jusqu'au XII siècle au moins. Si le Moyen Âge affectif apparaît tellement chrétien - alors même que l'on sait que la christianisation des mœurs et des corps des laïcs est la grande affaire de l'Église grégorienne et postgrégorienne uniquement - , c'est là non seulement un «effet de source» mais aussi une conséquence des structures normatives des sociétés médiévales. C'est bien de la nature même du message chrétien, et donc du rapport chrétien au monde, que découle la primauté d'un discours moral concernant l'usage de l'affectivité et du corps en société ${ }^{60}$, laquelle construit également une introspection selon une perspective sotériologique. Aussi, la plupart des sources, écrites dans un langage et «encodées » ${ }^{61}$ dans des catégories chrétiennes, nous renseignent-elles avant tout sur la vie affective selon les normes chrétiennes. Nous retrouvons ce langage et ces catégories aussi bien dans les textes théoriques sur les passions que dans les textes d'intention mystique, pédagogique, rhétorique ou politique, qui proposent l'émotion - ou y ont recours - comme un moyen, un instrument pour atteindre leur but ${ }^{62}$. Les destinataires de ces discours sont alors la plupart du temps des moines, des clercs et des moniales ou, dans une moindre mesure, des laïcs parmi lesquels on compte avant tout les aristocrates. En revanche, comme pour tant d'autres sujets, nous ne savons quasiment rien de l'affectivité des paysans. À partir du XII ${ }^{\mathrm{e}}$ siècle, la diversification des sources et l'essor de l'écrit vernaculaire permettent de dessiner une image de plus en plus nuancée des structures affectives des univers laïcs (des guerriers, de la culture courtoise, des couches diverses de la société urbaine), bien que l'empreinte chrétienne demeure marquée, sinon dans les enjeux explicites des textes, du moins dans la nomenclature et les normes qui les sous-tendent. Ainsi par exemple, nous ne pouvons cerner les significations possibles des pleurs des laïcs, hommes et femmes, dans les sources narratives, que si nous déchiffrons d'abord les normes chrétiennes régissant à la fois les pratiques des larmes et leurs représentations. Ce constat nous oblige à repartir au cœur du système affectif chrétien, à ce qu'on peut appeler avec Peter et Carol Stearns l'émotionologie hégémonique de l'Occident médiéval, notion qui permet de désigner «les attitudes ou normes qu'une société ou un groupe défini de la société entretient envers les émotions fondamentales et leur expression appropriée; les manières dont les institutions

60. Souligné également par C. CASAgrande et S. VeCChio, «Les théories des passions dans la culture médiévale», dans D. BoQuet et P. NAGY éd., Le Sujet des émotions..., p. 107-122.

61. Voir Codierungen von Emotionen im Mittelalter/Emotions and Sensibilities in the Middle Ages, I. Kasten éd., Berlin/New York, 2003 (Trends in Medieval Philology, 1).

62. Pour une réflexion sur les passions au Moyen Âge, voir C. Casagrande et S. Vecchio, «Les théories des passions »,loc. cit. 
reflètent et encouragent ces attitudes dans les comportements humains ${ }^{63}$, afin d'en explorer les dispositions vers l'incarnation de l'affect.

Le lien entre christianisme et émotions, la Passion et les passions, se noue au cœur du message évangélique, qui fait de la réflexion sur les émotions et le corps le centre même de son anthropologie ${ }^{64}$. En effet, les dogmes de l'Incarnation et de la Passion impliquent d'emblée une relation corps-âme et corps-émotion: Dieu s'est incarné par amour des hommes, et pour cette même raison il a souffert la Passion dans son corps. Le Dieu chrétien est donc tout affect, entretenant avec les hommes un lien d'amour, au point de faire souffrir son Fils pour eux. Ce lien est on ne peut plus charnel et il s'actualise jour après jour dans le rituel eucharistique. L'affection divine pour l'homme se manifeste également d'une manière très concrète et corporelle à travers les récits et les représentations de l'Incarnation et de la Passion, mais aussi par imitation dans les vies de saints. L'imitatio Christi, mot d'ordre de la perfection aux derniers siècles du Moyen Âge, passe alors par des gestes, voire des performances, affectifs et corporels ${ }^{65}$. Les modèles sont anciens, si l'on pense aux martyrs des grandes persécutions romaines, qui prouvaient l'ardeur de leur amour de Dieu par l'impassibilité de leur chair. Ainsi, l'émotion tire sa puissance du fait même qu'elle est incarnée, reliée au corps : loin de témoigner seulement de la tentation dualiste âme-corps, l'enracinement de l'affectivité dans la chair, pour le meilleur et pour le pire, se donne à lire comme une donnée anthropologique fondamentale de l'époque.

Du fait que l'Église, institution dominante de l'Occident médiéval, fonctionne comme une «bureaucratie du sens » ${ }^{66}$, les potentialités de ce message central de la religion chrétienne sont constamment réinvesties, réinterprétées, rejouées. Toutefois, l'essentiel des potentialités contenues dans l'Incarnation, ce mythe originel du christianisme, se déploie entre l'époque carolingienne et la grande réforme dite «grégorienne » de la société chrétienne. C'est à la faveur du processus d'identification de la société occidentale avec la christianitas ${ }^{67}$, puis quand société et Église deviennent coextensives lors de la réalisation du projet grégorien, qu'advient progressivement la civilisation de l'émotion incarnée.

63. P. N. Stearns et C. Z. Stearns, «Emotionology», loc. cit., p. 813.

64. Voir à ce sujet E. CocciA, «Il canone della passione. Il pathos di Cristo tra antichità e medioevo », dans D. Boquet et P. NAGy éd., Le Sujet des émotions..., p. 123-161.

65. D. Boquet et P. NAGY, «L'efficacité religieuse de l'affectivité dans le Liber (passus priores) d'Angèle de Foligno», dans $I l$ Liber di Angela da Foligno : temi spirituali e mistici, D. Alfonsı et M. Vedova éd., Spolète, 2010, p. 171-202.

66. L'expression est de M. Gauchet, Le Désenchantement du monde, Paris, 1985, p. 103 et sq. Pour notre sujet voir aussi, du même auteur, La Condition historique, Paris, 2003, particulièrement les chap. IV-V, p. 79-130.

67. Voir P. NAGY, «Les notions de christianitas et chrétienté et leur sens spatial. Abbon de Saint-Germain et la spatialisation de la christianitas», Médiévales, 49, 2005, p. 121-140. 
Cette émotion incarnée en Occident se construit dans la tension créée par le regard ambivalent du christianisme vis-à-vis du corps et de l'émotion. Dans l'Antiquité tardive, le péché s'est progressivement lié à la chair, et les vices aux passions, alors même que corps et amour sont indispensables à l'Incarnation et à la Passion. Si les passions apparaissent le plus souvent dangereuses pour le salut, la Passion devient le modèle de vertu suprême, du sacrifice de soi pour suivre le Christ. Animée par une tension verticale, la pensée médiévale de l'affect est moralement orientée selon l'axe passion-salut ${ }^{68}$ : toute passion, par sa nature ou par son usage, devient soit salutaire, soit nocive au salut. Cette tension structure les usages de l'affectivité, tant des émotions que des sentiments. Dans un premier temps, à l'inverse d'un véritable dualisme, la culture chrétienne du Moyen Âge occidental montre une forte tentation - du temps des Pères du désert au $\mathrm{XI}^{\mathrm{e}}$ siècle - à spiritualiser le matériel et plus précisément le charnel ${ }^{69}$. L'enjeu est de transformer par l'affectivité ce qui tire l'homme vers le bas, la terre, en élan vers le haut, le ciel. Dans un second temps, à partir du XII ${ }^{\mathrm{e}}$ siècle, l'on constate un mouvement inverse d'incorporation ou d'incarnation, l'investissement du corps par le spirituel, dont l'affect devient le véhicule. De ce mouvement d'incarnation, les témoins les plus connus sont François d'Assise et les figures mendiantes, cisterciennes ou laïques de la mystique féminine qui connaissent leur apogée entre le XIII et le XIV ${ }^{\mathrm{e}}$ siècle. Enfin, à la faveur de la renaissance du XII ${ }^{\mathrm{e}}$ siècle et de la redécouverte de l'aristotélisme, s'amorce une troisième voie qui vient détacher le lien de l'affect avec le corps de la logique et de la tension uniquement verticales. C'est au sein de ce mouvement général, qui investit progressivement le domaine du terrestre d'une valeur autonome, que le corps, imprégné de l'affect, voire de la passion, se retrouve pris dans des logiques de plus en plus dégagées du céleste. C'est dans ce contexte que se développe la doctrine de la pénitence qui accorde un rôle fondamental à la contrition, cette émotion qui rend efficace l'acte de pénitence, précédée dans la théologie monastique par la componction. Cette nouvelle efficacité émotionnelle prend forme dans le cadre conjoint de l'élaboration de la théologie sacramentelle au XII ${ }^{\mathrm{e}}$ siècle et de la promotion sociale de la confession auriculaire autour de Latran IV. Ce cadre offre une place, qui croît dans les derniers siècles du Moyen Âge, à la réflexion sur les émotions qui se retrouvent identifiées en partie à des vices, en partie à des vertus salutaires . De plus, avec le développement de la dévotion mystique, qui transpose dans le cadre intime l'efficacité religieuse des émotions définie pour le cadre sacramentel,

68. C. Casagrande et S. Vecchio, «Les théories des passions...», p. 109.

69. J. BASCHET, «Âme et corps dans l'Occident médiéval: une dualité dynamique, entre pluralité et dualisme», Archives de Sciences Sociales des Religions, 112 (2000), p. 5-30. 
on assiste à une appropriation par l'affectivité de l'efficacité sacramentelle, sous l'effet de ce que nous appelons l'émotif sacramentel ${ }^{70}$.

Les vecteurs de l'affectivité morale et l'usage des corps caractéristiques du christianisme sont loin de tout expliquer dans la relation entre émotions et corps au Moyen Âge. Ils sont toutefois toujours présents dans les sources, de manière explicite ou sous-jacente. Prendre en compte la centralité et la complexité de ces liens était donc impératif, et a largement déterminé la décision de mettre le corps affectif, l'émotion incarnée au cœur de nos préoccupations. Car, si notre projet avait pour but de mettre au jour les relations multiformes entre corps et émotions, c'est aussi parce que, paradoxalement, le corps est encore négligé dans l'histoire des émotions.

Les textes qui suivent questionnent la nature des liens entre émotion et corps au Moyen Âge, ainsi que leurs modalités d'expression, de mise en scène et de signification. Tous le font en intégrant les réflexions épistémologiques qui rendent possible aujourd'hui une histoire des émotions. Dionysios Stathakopoulos interroge dans la rhétorique des prédicateurs tardo-antiques le rapport entre les corps des mendiants lépreux et les émotions, éprouvées tant par ces derniers que par les spectateurs, rappelant ainsi l'étroite dépendance des émotions avec les arts du langage. Les articles de Nira Pancer et de Barbara Rosenwein proposent deux typologies possibles pour guider la lecture du lien corps-émotion. Nira Pancer distingue différents types de gestes somatiques. À une extrémité, se trouve le lapsus corporis, le corps trahissant l'émotion sans le consentement du sujet. À l'autre extrémité, le geste émotionnel public, ritualisé et mis en scène, relève de la performance, selon des règles culturelles connues, ou encore du performatif dont le sens dépasse le cadre convenu et se déploie: ainsi, un sourire ou des larmes, pouvant signifier un défi ou une menace, opèrent une transformation du lien social. Barbara Rosenwein étudie, quant à elle, trois communautés émotionnelles médiévales à des époques différentes pour montrer les degrés d'intensité du lien entre expression émotionnelle et corps, de la cour neustrienne où l'émotion est tenue loin du corps jusqu'à l'entourage de Margery Kempe pour qui toute émotion prend une forme incarnée. Cette puissance de l'émotion incarnée est dominante, qu'il soit question d'un geste mettant en scène l'émotion, ou d'un raisonnement émotionnel dont le poids moral permet de transcender les faits bruts des gestes du corps, comme dans le cas des nonnes de Watton convoqué par Damien Boquet. Guillemette Bolens, pour sa part, s'interroge sur le défi interprétatif et le jeu épistémologique lancés par Chaucer autour de la représentation narrative de l'émotion incorporée. S'attachant à la question épineuse de la représentation

70. D. Boquet et P. NAgy, «L'efficacité religieuse de l'affectivité... », loc. cit. 
de l'émotion, Robert Marcoux nous montre que des multiples figurations de la tristesse du Christ, seule celle par la face voilée permet de lui attribuer une signification positive. Enfin, l'analyse proposée par Klaus Oschema des gestes de toucher apportant la conciliation dans le duel judiciaire à la fin du Moyen Âge témoigne une nouvelle fois de ce que les émotions qui se concrétisent dans les gestes du corps agissent souvent dans les sources comme des facteurs de changement, comme des émotifs au sens où l'entend W. Reddy.

Damien Boquet - Université d'Aix-Marseille I, Département d'histoire, 29, avenue Robert Schuman, 13621 Aix-en-Provence CEDEX 1, France

Piroska NAGY - Université du Québec à Montréal, Département d'histoire, Case postale 8888, succursale Centre-ville, Montréal (Québec) H3C 3P8, Canada 